\title{
Positron Emission Tomography of the Airway Distribution of Intranasal Challenge Solutions
}

\author{
L. Conejero ${ }^{1}$, M. L. Soto ${ }^{1}$, J. J. Vaquero ${ }^{1}$, M. L. Baeza ${ }^{1}$, E. Lage ${ }^{1}$, I. Varela-Nieto ${ }^{2}$, M. Desco ${ }^{1}$, J. M. Zubeldia \\ ${ }^{1}$ Hospital Gregorio Marañón, Madrid, SPAIN, ${ }^{2}$ Instituto Investigaciones Biomédicas Alberto Sols. CSIC-UAM, Madrid, \\ SPAIN.
}

RATIONALE: Intranasal administration is one of the main routes of allergen challenge in mouse models of airway disease. Although it is widely used, it is not well established the amount of allergen that reaches the lung or is lost to the gastrointestinal tract. The local distribution of the challenge solution within the airways is also unknown. The aim of this study was to assess the distribution immediately after intranasal delivery using a Positron Emission Tomography scanner (PET).

METHODS: Fifteen BALB/c mice, 15-20 weeks-old, were studied. Thirty microliters containing 300mCi of 2-deoxy-2-[18F] fluoro-D-glucose (FDG) were administered intranasally. Mice were anesthetized and imaged during $30 \mathrm{~min}$ in a dedicated small-animal PET scanner (rPET, SUINSA).

Images were reconstructed by the 3D-Filtered Back Projection (3D-FBP) method and Regions of Interest (ROIs) were drawn on coronal and axial sections to obtain quantitative data of lung activity. Dynamic curves were obtained at $40 \mathrm{sec} /$ frame and the value at initial time was extrapolated after exponential fitting.

RESULTS: Of the total dose administered, 31.2\%68.5\% (mean6SD) reached the lower airways. A comparable percentage was delivered to each lung with a 51612\% estimate located into the right lung. The tracer distribution was homogeneous within the areas reached, as previously assessed visually.

CONCLUSION: PET imaging confirms that intranasal route is a suitable technique for delivering allergenic fluids into the lower airways. However, it should be taken into account that, in average, only one third of the administered dose reaches the anatomical target.

Funding: FIS 01/0598 and Foundation SEAIC 